\title{
Cognitio Juris
}

\section{EL DERECHO DESDE LA SOCIOLOGÍA Y LOS MOVIMIENTOS CULTURALES}

Juan de Dios González Ibarra

\begin{abstract}
RESUMO
Desde la perspectiva de la crisis del derecho producto de su incapacidad para ser el gran organizador nacional y global, lo primero por la presencia de culturas jurídicas líquidas o débiles y, lo segundo, por la poca eficacia internacional de la ONU por la fuerza monopólica de Estados Unidos, aquí se buscan alternativas para construir fraternidades nacionales y mundiales.
\end{abstract}

Palavras- chave: Derecho, sociología, cultura, movimientos culturales, fraternidad.

\section{THE LAW FROM SOCIOLOGY AND CULTURAL MOVEMENTS}

\begin{abstract}
From the perspective of the crisis of the law product for their inability to be the great organizing national and global, first by the presence of liquid or weak legal cultures and, second, by the little international effectiveness of de NU by the monopoly force of United States, we are looking for alternatives to build global and national fraternities.
\end{abstract}

Key-words: Law, sociology, culture, culture movements, fraternity.

1. El objeto de la sociología es el estudio de la relación entre el individuo humano como ser con una personalidad única e irrepetible universalmente y la sociedad en la cual se encuentra ubicado,104 en cuanto a la sociología jurídica en lo general estudia la relación entre el derecho y la sociedad valorando la eficacia y eficiencia del primero conforme parámetros establecidos previamente.

El maestro español Gregorio Robles reconoce que se mencionan como precursores de la sociología a Montesquieu y Tocqueville, con el antecedente de la llustración definida como el movimiento intelectual europeo en el que los ilustrados creían en el poder liberador de la razón humana y en el progreso social, en 1784 "Kant se refiere a ella como el momento en que la humanidad alcanza la mayoría de edad y rompe con todo tipo de tutela previa al tomar plena conciencia de su propia libertad".105

Sin embargo como ciencia con objeto y método propio empieza con Augusto Comte y Durkheim, siendo "la sociología un producto intelectual típico de la mentalidad positivista",106 el primero tomó como modelo a la física y sus leyes causales, por lo que Comte la llamó en un principio física social, adjudicándose la pretensión de ser la ciencia social que podía explicar la totalidad de la epistemología científica de la realidad social, lo que generaría un enfrentamiento con el estudio centenario del derecho o jurisprudencia, así los jurisconsultos no aceptaron someterse a la joven ciencia, y desarrollaron la doctrina general del derecho igualmente positivista, al que seguiría por el camino de la codificación napoleónica el positivismo legalista verdadero -según Roblesiusnaturalismo encubierto. La sociologización del pensamiento jurídico se realizó principalmente con tres vertientes: la primera encabezada por Jhering o de la jurisprudencia de intereses, la segunda de vocación empirista investigando los hechos jurídicos con Nussbaum a la cabeza y, la tercera de Ehrlich y Weber que busca la sustitución de la dogmática jurídica por la sociología del derecho, considerando Robles a los jurisconsultos mencionados como fundadores de la sociología jurídica.

El abogado y politólogo colombiano Mauricio Villegas, a quien seguiremos en este trabajo, menciona que el prestigiado economista Jeffrey Sachs "sostuvo que los grandes desafíos del mundo estaban relacionados con la incapacidad del derecho de responder a las demandas de regulación social",107 siendo según él actualmente los dos grandes retos o paradojas, el construir el Estado constitucional para evitar el abuso de poder, y el segundo, formar un sistema internacional que no permita "los desafueros del mercado y de las acciones de los Estados soberanos". Esto nos llama a la reflexión a los estudiosos del derecho de su eficacia como gran armonizador social.

Es interesante establecer una relación de la afirmación anterior con la de Carlos Granés, quien sostiene que las

104 BATIFOL, Henri, Filosofía del derecho, Conaculta-Publicaciones Cruz, México, 1995, p. 44.

105 MAGEE, Bryan, Historia de la filosofía, Planeta, México, 1999, p. 134.

106 ROBLES, Gregorio, Sociología del derecho, Civitas, Madrid, 1997, pp. 23-28.

107 GARCÍA VILLEGAS, Mauricio, Sociología y crítica del derecho, Fontamara, México, 2010, p. 9. 


\section{Cognitio Juris}

REVISTA JURÍDICA COGNITIO JURIS I JOÃO PESSOA - ANO \|I - NÚMERO 4 - ABRIL 2012 | ISSN 2236-3009

revoluciones políticas del siglo pasado al final terminaron en derrotas, mientras que por diferencia las culturales con los futuristas, dadaístas, surrealistas, yippies y otros permitieron que "sus batallas por transformar la vida resultaran fructíferas".108 Entonces bajo esta lógica ¿podemos concebir que el camino del cambio eficaz está en la cultura?, ¿qué es el desarrollo de una cultura jurídica amplia y sólida un mejor camino para construir instituciones, ciudadanía autoroganizada para una mejor convivencia social?

Bajo las anteriores hipótesis desarrollaremos este trabajo, buscando la interdisciplinariedad y la crítica propositiva, con el contexto de un México inequitativo con el cincuenta por ciento de su población sumida en la pobreza o miseria, elevada corrupción, impunidad, desconfianza, opacidad, además de encontrarse sumido en una guerra que en cinco años nos ha costado cincuenta mil muertos, la mayoría jóvenes.

La afirmación de que para el avance del derecho se requiere de más crítica y sociología jurídica es afortunada, con los referentes de Marx, Émile Durkheim y Max Weber, lo que se señala con la afirmación de García Villegas "Paradójicamente, nunca antes hubo tantas razones para preocuparse por las instituciones que nos gobernaron y por el derecho que no s reguló. La inequidad, la violencia y la inseguridad, por sólo mencionar tres de los más esenciales males que el derecho está llamado a remediar, no sólo no se atenuaron sino que se agravaron".

Las estadísticas indican que "Hoy en día la mitad de la población del mundo vive en medio de la miseria, con menos de dos dólares diarios. En América Latina había más gente pobre a fines de los 90 que en 1980. Incluso en Estados Unidos, el país más rico y poderoso del planeta, la población que vive bajo la línea de pobreza pasó de 25 millones en 1970 a 37 millones en el 2007".

Por lo que concierne a la violencia "si en el siglo XVIII se calcula que murieron unos cuatro millones de personas en guerras, en el siglo XX murieron cerca de 200 millones", por lo que respecta a la amenaza ecológica "los problemas del calentamiento global parecen hoy insolubles y ello debido, en buena parte, a la falta de voluntad de los países ricos que albergan el 20\% de la población mundial, pero consumen el $80 \%$ de la energía".109

Por lo anterior podemos estar de acuerdo con García Villegas que la sociología del derecho ha tenido enfrente como adversarios a la dogmática y teoría general del derecho. Analizando a Bourdieu se establece que el derecho es un campo social en donde se lucha para apropiarse del poder simbólico, mismo que poseen los textos jurídicos con un grado de autonomía relativa, así quien controla el derecho tiene también el control social.

La crítica del derecho desde la sociología nos proporciona la perspectiva enriquecedora de poder contemplar a este campo con la visión del contexto social, pudiendo valorar la eficacia y eficiencia del mismo en relación con su fin de gran organizador social para poder convivir en la paz y armonía.

2. Derecho y movimientos culturales. Para empezar a desarrollar este apartado nos apoyaremos en el pensamiento de mí querido maestro Bolívar Echeverría, quien en una obra relacionada con este campo110 parte de la que él llama la dimensión cultural, continúa con las diversas definiciones de cultura y concluye con la problemática actual referente al estudio de ella y su historia. Formula su propia definición desde un marco teórico marxista así para él "la cultura es el momento autocrítico de la reproducción que un grupo humano determinado, en una circunstancia histórica determinada hace de su singularidad concreta; es el momento dialéctico del cultivo de su identidad"111

Reconociendo que el término cultura viene desde Roma, se refiere al cultivo "de la humanitas, de aquello que distingue al ser humano de los demás seres".112 Establece la sana distinción entre civilización y cultura, señalando por nuestra parte que la primera se relaciona con la razón instrumental o empírica, es pragmática y utilitarista, por diferencia la cultura establece una íntima relación humanística o de superación de la condición del hombre y la mujer en relación con el espíritu, -entendiendo a éste como la capacidad de pensar, reflexionar y actuar conforme determinados valores internalizados por el individuo y aquellos que colectivamente imperan en una sociedad-.

Históricamente se presenta esta disputa entre civilización y cultura en la lucha entre clase noble y burguesía, con lo que la nobleza es criticada, aparte de por ser improductiva por sus modales, en donde éstos hacen la crítica de las meras formas cortesanas superficiales, así "los intelectuales pequeñoburgueses se atreven a 'mirar por encima del hombro', en el terreno del espíritu, a los aristócratas, cuyo comportamiento vacío y frívolo desdice la superioridad jerárquica que ostentan en lo social y lo político".113

108 GRANÉS, Carlos, El puño invisible. Arte, revolución y un siglo de cambios culturales, Taurus, México, 2011, p.14.

109 Opus cit., pp. 10-11.

110 ECHEVERRÍA, Bolívar, Definición de la cultura, FCE-Ítaca, México, 2010, pp. 17- 41.

111 Opus cit., pp. 163-164.

112 Opus cit., p. 28.

113 Ibídem, p. 29.

64 I Cognitio Juris 


\section{Cognitio Juris}

REVISTA JURÍDICA COGNITIO JURIS I JOẼO PESSOA - ANO \|I - NÚMERO 4 - ABRIL 2012 | ISSN 2236-3009

Así el reto se ha establecido desde la cultura, lo que Karl Max lo contemplará desde lo económico e ideológico y Antonio Gramsci desde la praxis del dominio de clase revestido de consenso. En la llustración la creatividad cultural es libre y desinteresada, mientras que la de la civilización persigue fines lucrativos, reconociendo las influencias de René Descartes (1596-1650), Isaac Newton (1643-1727) con su método analítico de la ciencia de la física como modelo a aplicar en todos los campos, hasta que J. G. Droysen (1808-1884) distinguió entre explicación (Erklären) y comprensión (Verstehen) para fundamentar el método de las ciencias histórico-sociales, cuya función sería comprender, en contraposición a las ciencias físico-naturales que intentan explicar por medio de leyes causales, con lo cual podemos hablar de ciencias de la naturaleza y ciencias del espíritu,114 criterio que respetaría W. Dilthey (1833-1911) en su libro Introducción a las ciencias del espíritu.

Desde la filosofía para Ortega y Gasset la cultura es una tabla de salvación para que el ser humano no se hunda en la vacuidad de la vida cotidiana,115 así nos recuerda que la máxima de "conócete a ti mismo", sirve para llegar a ser aquello que podemos llegar a ser gracias a los dones que todos poseemos y que nos viene intrínsecamente desde nuestra propia naturaleza116 humana que tiende al saber.

En el Romanticismo, que surge como reacción contra el intelectualismo cartesiano de la razón instrumental que venía de la llustración, la cultura es producto de la creatividad del genio creador, mientras que la civilización viene de la actividad intelectualmente calificada, aquí la práctica técnica cobra relevancia.

En relación con nuestro país para uno de los más ilustres intelectuales mexicanos el maestro Ignacio Pichardo Pagaza, formado en derecho por la UNAM y en administración pública y economía en el prestigiado Dartmouth College de New Hampshire y en la Escuela de Economía de Londres, afirma que "el problema número uno de México consiste en tener una población insuficientemente educada. Por tanto, la prioridad más alta es buscar y conseguir la educación de calidad para todos".117

3. Gestión cultural. Si conforme hemos apreciado es indispensable impulsar una cultura sólida en nuestro país para salir del estado lamentable118 en el que nos encontramos de corrupción, inseguridad, analfabetismo lector (dos libros por años es nuestro promedio de lectura, contra veinte en Europa; Carlos Monsiváis señaló que en México generalmente los universitarios sólo leen sus libros técnicos) y el analfabetismo científico (maestros y doctores que desconocen metodologías, epistemologías y lógicas) y analfabetismo humanístico ante la ignorancia de literatura, pintura, música clásica, escultura, aquí Pichardo Pagaza apela a realizar una revolución por la educación y los valores, luego entonces en consecuencia lógica es en este campo de la gestión cultural en donde debemos dirigir nuestros esfuerzos nacionales.

En lo general la gestión cultural es parte de la gobernanza que integra la acción del Estado con la de la ciudadanía autoroganizada, consiste en dirigir nuestros esfuerzos hacia el cultivo de las cualidades del ser humano para superar nuestra condición humana y a nuestra comunidad.

Nos apoyaremos críticamente, por encontrar en la obra119 de la doctora en derecho María Elena Moreira una visión economicista, ella es periodista ecuatoriana con estudios en derechos humanos de la Universidad de Lund, Suiza, quien afirma que "la gestión cultural es la respuesta de un mundo globalizado a un nuevo concepto de cultura y al espacio cada vez más amplio y complejo que la actividad cultural ocupa en la sociedad. Este nuevo rol de la cultura exige la formación de individuos capacitados para encarar el manejo de las distintas facetas del proceso cultural".

Resalta su importancia al señalar que el sector económico cultural se incrementa conforme el mayor desarrollo económico del país, así en los Estados Unidos, en el que se incluye el entretenimiento, es el primer sector de exportación, superando a la industria pesada, militar o aeronáutica representando el $2.5 \%$ del PIB, en Francia es del $3.7 \%$ y Brasil apenas el $0.8 \% .120$

Existen dos modelos dominantes en este campo: el centralista europeo como el francés o el de Alemania que subsidia y administra el $80 \%$ de la cultura de ese país y el norteamericano en el que el Estado no interviene mayormente, dejando las políticas de impulso a la cultura en manos del equivalente a nuestros municipios o

114 URSUA, Nicanor, AYESTARÁN, Ignacio y GONZÁLEZ, Juan de Dios, Filosofía crítica de las ciencias sociales y humanas. Historia, metodología y fundamentación científica, Ediciones Coyoacán, México, 2004, p. 68.

115 FERRATER MORA, J, Diccionario de filosofía, Tomo I, Ariel, Barcelona, 2001, p. 764.

116 ORTEGA Y GASSET, José, ¿Qué es filosofía?, Espasa Calpe, Madrid, 1995, pp. 83-86.

117 PICHARDO PAGAZA, Ignacio, El camino de México, una revolución por la educación y los valores, Miguel Ángel Porrúa, México, 2011, p. 13.

118 Zygumnt BAUMAN nos señala que hay sociedades líquidas o débiles institucionalmente y sólidas o fuertes.

119 MOREIRA, Elena, Gestión cultural. Herramienta para la democratización de los consumos naturales, Longseller, Argentina, 2003, p. 9.

120 Opus cit., p. 77. 


\section{Cognitio Juris}

REVISTA JURÍDICA COGNITIO JURIS I JOÃO PESSOA - ANO \|I - NÚMERO 4 - ABRIL 2012 | ISSN 2236-3009

counties o los individuos. Así en EE. UU. "sólo uno de cada diez dólares invertidos en la cultura proviene del Estado, mientras que el $75 \%$ de los fondos proviene de contribuciones individuales, ni siquiera de corporaciones o fundaciones".121

Señala Elena Moreira que es a partir de los años 60 y 80 en que la gestión cultural forma parte de las políticas públicas de los Estados, generando la necesidad de formar especialistas en este campo. Por nuestra parte sostenemos que se requiere que aplicando interdisciplinariamente los conocimientos y saberes de la administración, bellas artes, tecnología, ciencias sociales y humanidades, obtengamos los mejores resultados con los recursos disponibles siempre escasos ante las necesidades culturales.

Conforme lo anterior "su objetivo principal es establecer canales que promuevan la participación de las personas en la dinámica cultural, la cual, a la vez, retroalimenta y estimula los fenómenos creativos y los hábitos culturales"... tiene la responsabilidad de favorecer el desarrollo cultural en su calidad de mediador entre los fenómenos expresivos o creativos y los públicos que conforman la sociedad".122

Encuentra cuatro modificaciones recientes en este campo: a) ampliación del concepto de cultura por razones filosóficas, sociales, políticas y legales; b) las crisis de las apreciaciones políticas y de desarrollo desde los años 60; c) "la necesidad de políticas culturales que gestionen ámbitos más allá de la cultura artística, la tradicional y el patrimonio" y d) nuevos cambios teóricos y prácticos entre economía y cultura.

3.1. Los cambios en el concepto de cultura. A partir de la diferencia griega entre estado de naturaleza o natura y estado de la cultura, con las discusiones subsecuentes de cultura contra naturaleza, como en los filósofos cínicos que "proclamaron su oposición a todo lo que no fuera la simplicidad natural, considerándose la cultura como un signo de corrupción y decadencia"123, o si es superior el estado de cultura al de naturaleza o viceversa por ejemplo en la obra de Juan Jacobo Rousseau de Emilio o De la educación, en donde se afirma que el ser humano es bueno por naturaleza y que la civilización lo pervierte, estamos ante el mito del "buen salvaje".

Apreciamos que cada época construye su propio significado de cultura, así en Roma la cultura estará dominada por aquel pueblo que las legiones romanas conquistaron: el griego; posteriormente con el triunfo de la religión cristiana se impondrá la cultura judeocristiana, con las guerras religiosas al surgir el rompimiento con el papa por parte de Enrique VIII de Inglaterra el protestantismo se imponen, con la posterior visión de Max Weber de la importancia de esta religión para el desarrollo del capitalismo precisamente partiendo de ese país con su Revolución Industrial, el racionalismo cartesiano junto con el lluminismo plantean el dominio de la razón sobre el dogma y la fe, el Romanticismo invoca al espíritu de los pueblos que es el que construye lenguajes y sistemas jurídicos y sociales, la modernidad presenta al dominio de la razón capitalista instrumental en la que prevalece el mito de que la razón y el mercado dará la felicidad a la humanidad y, finalmente, la posmodernidad que en reacción contra la anterior cuestionará supuestos logros que no han favorecido más que a una pequeña minoría y, en donde, se ampliará la brecha entre quienes tienen poder económico, político y capital cultural, aunado al problema ecológico y el de las armas atómicas que amenazan a la humanidad, así las promesas de la modernidad hoy se muestran falsas con los doscientos muertos en las guerras del siglo pasado en comparación con 16 millones del siglo XIX.

El reto de este siglo es construir una cultura de paz universal, conforme lo previó Emmanuel Kant en su obra De la paz perpetua, de otra manera es difícil que podamos sobrevivir como especie humana. Aparte de la anterior el desarrollo de una cultura ecológica forma parte de la lucha por la sobrevivencia de la humanidad, así el Informe de la ONU 2010 es por demás alarmante al respecto pues muestra que es casi nada lo que se está realizando en contra del calentamiento y de la contaminación. Los dos grandes gigantes Estados Unidos y China, quienes son los que más contaminan tienen políticas débiles en este campo, explicables porque el primero no desea dejar su papel de primera potencia del mundo y, el segundo para no detener su veloz carrera tecnológica y financiera, sin embargo es el destino de todos lo que está en grave riesgo.

Si desde las humanidades se afirma que la calidad humana tiene implícitamente una dignidad única por ser fin y no medio cualquier persona del mundo, hoy con el peligro ecocida124 también la naturaleza debe tener un reconocimiento prioritario, recordando que es la Amazonia el gran pulmón del mundo.

En México en lo particular, aparte de lo señalado antes, requerimos llevar a cabo las revoluciones culturales 125

121 lbídem, p. 82.

122 Opus cit., pp. 13-14.

123 FERRATER MORA, José, Opus cit., p. 763.

124 Es el doctor Luis TAMAYO, formado en Alemania y Francia, intelectual del Centro de investigaciones y Docencia en Humanidades de Morelos, el máximo especialista mexicano al respecto.

125 GOLDMANN, Lucien, La creación cultural en la sociedad moderna, Ediciones Coyoacán, México, 2008, p. 7. El intelectual mexicano David RINCÓN PÉREZ atribuye, acertadamente en nuestro criterio, la paternidad del 


\section{Cognitio Juris}

de lectura para rebasar con mucho los dos libros anuales como promedio nacional, la de cambiar nuestro analfabetismo científico y humanístico desde el impulso de las ciencias del espíritu, humanidades 126 y duras o de la naturaleza, ética para combatir la corrupción y jurídica en contra de la inseguridad, impunidad y opacidad contando para esto con el apoyo de la cultura jurídica sólida e instituciones de igual manera, aquí la nos ayudan la sociología jurídica, epistemología y nuestra capacidad de reflexión, para pasar de una sociedad líquida a una sólida con paz y sin la guerra que ha ocasionado más de cincuenta mil muertos, la mayoría de ellos jóvenes de origen campesino que no tuvieron, muchas veces opciones laborales, ni oportunidades culturales y educativas.

concepto de cultura a los romanos y se apoya en CICERÓN quien "hablaba de cultura animi, el cultivo del espíritu".

126 CEBALLOS GARIBAY, Héctor, La sabiduría de la novela, Fontamara, México, 2011, p. 14. Retomando al genio búlgaro-francés TZVETAN TODOROV concuerda con él en sus palabras "Coloco a la literatura por encima de la filosofía y la ciencia...Y no es sólo que la literatura participa de la elucidación del mundo, sino que también le agrega belleza y, de esa manera, lo hace mejor". 